\title{
Self-rated breast cancer risk among women reporting a first-degree family history of breast cancer on office screening questionnaires in routine medical care: The role of physician-delivered risk feedback
}

\author{
Robert Gramling, $M D^{1}$, David Anthony, $M D, M S c^{1}$, Emma Simmons, $M D, M P H^{1}$, and Deborah Bowen, $P h D^{2}$
}

\begin{abstract}
Purpose: We investigated whether risk-related feedback delivered by one's primary care physician is associated with self-ratings of risk among women found to have a first-degree family history of breast cancer on office screening questionnaires. Methods: Design: Mailed survey of women registered with the Cancer Genetics Network having a first-degree family history of breast cancer. Eligibility: Completion of primary care-based family history screening within the past year. Independent variable: presence of physician feedback about breast cancer risk. Dependent variable: self-rated breast cancer risk. Modifying variable: trust in one's doctor. Results: Three hundred one women met eligibility criteria ( $73 \%$ minimum response rate); feedback was associated with rating one's risk to be "high" in both crude and multivariate analysis. $\left(\mathrm{OR}_{\mathrm{adj}}=2.38 ; 95 \% \mathrm{Cl}=1.30,4.38\right)$. Higher levels of trust in the physician were associated in a dose-dependent fashion with the strength of association between feedback and self-rating one's risk to be high. Conclusions: Physician feedback following the identification of a first-degree family history of breast cancer appears to influence whether or not women categorize themselves to be at high risk and trust is an important modifier of this association. Genet Med 2006:8(10):658-664.
\end{abstract}

Key Words: Screening, genetics, primary care, risk perception, physician-patient communication

The United States Preventive Services Task Force (USPSTF) recommends that women "whose family history is associated with an increased risk for deleterious mutations in $B R C A 1$ or $B R C A 2$ genes be referred for genetic counseling and evaluation for BRCA testing" but to avoid referring women with lower-risk family histories because the harms outweigh the benefits ${ }^{1}$ Additionally, the USPSTF recommendation to begin mammography at age 40 years is strengthened for women having a first-degree family history of breast cancer. ${ }^{2}$ These and related recommendations reinforce the clinical use of family history in primary care practice for the purposes of shared decision-making about breast cancer prevention strategies, including pursuit of genetic testing. Despite the evidence suggesting that primary care physicians lack confidence in the assessment of heritable cancer risk, ${ }^{3}$ most of the decision-making about whether one's family history is clinically relevant or not will take place in the primary care setting. Little is known about

From the ${ }^{I}$ Brown Medical School, Providence, Rhode Island; ${ }^{2}$ Fred Hutchinson Cancer Research Center, Seattle, Washington.

Robert Gramling, MD, Center for Primary Care and Prevention, Memorial Hospital of Rhode Island, 111 Brewster Street, Pawtucket, RI 02860.

Submitted for publication April 7, 2006.

Accepted for publication June 26, 2006.

DOI: 10.1097/01.gim.0000237769.59166.ad whether, and how, handling of family history in primary care influences a patient's perceptions of her breast cancer risk.

The judgments that individuals make regarding their risk to adverse outcomes are central to influential theories of preventive decision-making and behavior. ${ }^{4-6}$ Risk-related information that arises within a trusted medical relationship is more likely to be internalized, and therefore shape such judgments, than messages from less trusted sources. ${ }^{4}$ In the context of primary medical care, many women have their family history solicited on office screening forms or via physician questioning. Soliciting cancer family history reinforces to the patient the clinical relevance of her genetics and cues the patient to consider whether her familial risk is medically significant. The feedback that a woman receives from her primary care physician may either reinforce or challenge existing beliefs that she holds regarding her personal breast cancer risk. The effect of such feedback may be especially strong if she has a trusting relationship with that doctor.

This study will examine whether physician-delivered breast cancer risk feedback to women who have reported a first-degree family history on office screening forms is associated with women's self-rating of her breast cancer risk. Furthermore, we evaluate the degree to which women's degree of trust in their regular physician modifies the relationship between receipt of cancer risk feedback and self-rated risk. 


\section{MATERIALS AND METHODS}

\section{Design}

We performed a cross-sectional survey using a mailed questionnaire from November, 2003 to February, 2004 among women registered with the Cancer Genetics Network (CGN). ${ }^{5-7}$

\section{Human subjects}

The development and pre-testing of the measures were approved by the Miriam Hospital Review Board for Protection of Human Subjects (Rhode Island).

The main study was approved by the Institutional Review Boards for Miriam Hospital (Rhode Island), the University of New Mexico, the University of California at Irvine, the University of Colorado Health Sciences Center, and the University of Utah.

\section{Setting}

The CGN ${ }^{7-9}$ is a federally-funded, collaborative project that aims to: provide infrastructure for studying the genetic basis of cancer susceptibility; understand the psychosocial, ethical, legal and public health issues related to genetic susceptibility to cancer; and investigate the best means for incorporating such information into medical care. One major objective of the CGN is to recruit individuals having a family history, but no personal history, of cancer in order to create a contact registry for future studies related to cancer susceptibility. All consenting participants complete a baseline telephone interview consisting of a four-generation pedigree and socio-demographic information. All enrollees agree to be approached for participation in future research studies and are contacted annually to update their baseline data.

The CGN comprises eight recruitment sites across the U.S. Half of these sites recruit participants via population-based methods and the other half use clinic-based recruitment strategies. ${ }^{9}$ This study was restricted to participants recruited to the CGN via population-based strategies to minimize the selection bias represented by recruiting individuals from highrisk cancer centers and genetic counseling programs. Population-based sampling covered a large geographic area and is very unlikely to have sampled women sharing the same regular physician. All CGN recruitment, interviewing and follow-up protocols were standardized across centers and approved by the site Institutional Review Board for protection of human subjects.

\section{Participants}

We mailed a cover letter, refusal card and survey to 585 women registered with the CGN who met the following criteria as established by the baseline telephone interview completed upon recruitment to the CGN: Twenty-five to 65 years of age, family history of breast cancer in a first-degree relative (FDR) and no personal history of a major cancer (i.e., any cancer other than non-melanomatous skin cancer). Final eligibility for this study included women having a regular source of medical care, having visited their regular doctor within the past year and having completed office screening forms soliciting family history information. These final criteria could be assessed only for those completing the study questionnaire. Our response rate calculations assume that all non-responders would have met these final eligibility criteria and therefore are likely to have biased our response rates downward.

\section{Data collection}

The 7-page questionnaire consisted of 34 multiple-choice items. For this study, we used the following variables: having a regular physician; last visit to that physician; completion of office family history screening forms; recalled feedback from the physician about cancer risk; trust in that physician; agreement with rating one's breast cancer risk to be "high"; perceived likelihood of developing breast cancer; and perceived severity of developing breast cancer. The remaining variables (age, education, breast cancer family history; and personal cancer history) were obtained from the CGN registry database that is updated annually.

Participants were provided postage-paid return envelopes and refusal postcards. Ten days after initial mailing, three attempts were made to contact non-responders by telephone over a one-week period. Those contacted could refuse participation or request another questionnaire. Those unable to be reached by telephone were mailed a second questionnaire within three weeks after the initial mailing.

\section{Measures}

All measures were designed explicitly for the goals of this study and underwent qualitative evaluation prior to arrival at the final items. All measures were subsequently pre-tested in the final instrument for question comprehension, response option comprehension and response burden among 25 women of diverse educational backgrounds.

\section{Risk feedback}

We categorized recalled risk feedback into three broad categories: direct feedback about breast cancer risk, discussion about cancer genetic referral, or neither of the above feedback types.

\section{Direct feedback}

Participants were asked the following, "Has your regular doctor talked with you about your chances of getting breast cancer someday? (words like "risk" and "likely" also refer to your chances)." Those who answered in the affirmative were considered to have received direct feedback. We chose not to ask about the specific content of the feedback (e.g., a physician telling a patient she is at high risk for cancer) since prior work ${ }^{10}$ suggests that recall of such specific details about patient-doctor communication can be misleading.

\section{Discussion about genetic referral}

Respondents were asked the following two items: "Has your regular doctor talked with you about seeing a genetic specialist because of your risk of breast cancer?" and "Has your regular doctor talked with you about genetic testing for your breast 
cancer risk?" Positive responses to either question constituted exposure to discussion about genetic referral.

Response options for all of the above independent variable items (direct risk feedback, discussion about genetic referral) included "Yes, in the last year," "Yes, more than 1 year ago," "Yes, I don't remember when," and "No." These items were adapted from a similar validated measure of patient interactions with their regular physician. ${ }^{11}$ To reduce recall error, we limited our timeframe to the last year for determination of exposure to risk feedback. Self-report about topics arising in the medical encounter have been demonstrated to be valid, ${ }^{10}$ particularly when the issue is relevant to the patient.

\section{Self-rated risk status}

All participants in this study had their family history solicited by their regular doctor. The purpose of such types of risk factor screening is to identify individuals at "high risk" for whom departure from standard clinical practices should be considered. Physicians tend to discuss risk with patients in subjective terms that reflect clinical thresholds (i.e., "high risk"). ${ }^{12}$ Therefore, we chose to measure self-rated risk in terms that are most likely to be affected by feedback in medical practice using wording advocated by Witte et al. ${ }^{6}$ We asked participants to respond to the following: "I am at high risk for getting breast cancer." Responses were recorded on a six-point Likerttype scale ranging from "strongly disagree" to "strongly agree." We dichotomized responses a priori to represent those self rating their risk as "high" ("strongly agree," "agree," and "somewhat agree") and "not high" ("strongly disagree," "disagree," and "somewhat disagree").

Cancer risk perception is often considered to be a continuous phenomenon that meshes constructs of perceived cancer likelihood and perceived severity of cancer in heuristic assessments of risk. ${ }^{13}$ We expect that clinical communication will influence self-categorization of risk (i.e., high risk) to a greater degree than self-ratings of risk on continuous scales. Therefore, a secondary aim of this study is to examine whether continuous measures of risk perception capture the phenomenon of physician-delivered risk feedback to a greater or lesser degree than self-categorization of "high risk" status.

For both perceived likelihood and perceived severity constructs, we used single-item measures having six-point scales that have been advocated in the risk perception literature. ${ }^{6,14}$ For perceived likelihood, we asked the following: "How likely do you believe it is that you will get breast cancer someday?"(Response options: 1 = No chance, $6=$ Certain to happen). For perceived severity, we asked the following: "Getting breast cancer would be a very serious problem." (Response options: $1=$ Strongly disagree, 6 = Strongly agree).

\section{Potential confounder/effect modifying variables}

We considered the following variables as potential confounders or effect modifiers of our main association between cancer risk feedback and self-rated risk status: age, education and presence of higher-risk family history characteristics. All eligible women had at least one FDR diagnosed with breast cancer. We defined high-risk family history characteristics in the method advocated by Scheuner et al. ${ }^{15}$ to be the presence of an affected male FDR, multiple affected FDRs, or an FDR with age of onset of 50 years or less.

\section{Trust}

Behavioral theory posits that the degree of trust in the source of risk information should influence the degree to which the recipient of the risk message internalizes the information to shape perceptions. ${ }^{4}$ We considered whether the degree of trust in one's physician modified the association between feedback and self-rated risk status. Each participant was asked the following previously validated question ${ }^{11}$ : "All things considered, how much do you trust your regular doctor?" Responses were recorded on a scale from 0, "Not at all," to 10, "Completely." For analyses of effect modification, the scale was categorized into the following quartiles based on observed distribution of responses: 0 to $7=$ "Low trust," $8=$ "Moderate trust," $9=$ "High trust," and $10=$ "Very high trust."

\section{Analyses}

\section{Crude and stratified comparisons}

Frequency and distributions of all study variables were assessed to determine appropriate statistical tests for continuous variables. Crude and stratified analyses were conducted for the association between cancer risk feedback and self-rated risk in order to investigate potential confounding or effect modification. Standard chi-square tests were used to identify statistically different proportions.

\section{Control of confounding}

We fit a multiple logistic model for all potential confounding variables and proceeded with a backwards model reduction procedure. The procedure involved inclusion of all covariates exhibiting at least a $10 \%$ change in effect on stratified analysis and removal if the absence of the covariate from the model resulted in $<10 \%$ overall change in the final adjusted measure of effect. Those factors exhibiting effect measure modification in crude analyses (i.e., trust) were considered as possible effect modifiers in adjusted analyses by stratified modeling.

For adjusted analyses of continuous dependent variables (i.e., perceived likelihood, severity), we fit a generalized linear model including covariates from the resulting logistic modeling of our main association. For perceived severity, we fit our model to a Poisson distribution of the inverse severity score because women's perceptions of cancer severity were substantially skewed in the more severe direction.

\section{RESULTS}

\section{Response rate}

Figure 1 demonstrates recruitment and eligibility for our final study sample. Of the 585 women meeting initial eligibility criteria in the CGN registry, 476 women returned a completed questionnaire. Three hundred one women met the final eligi- 

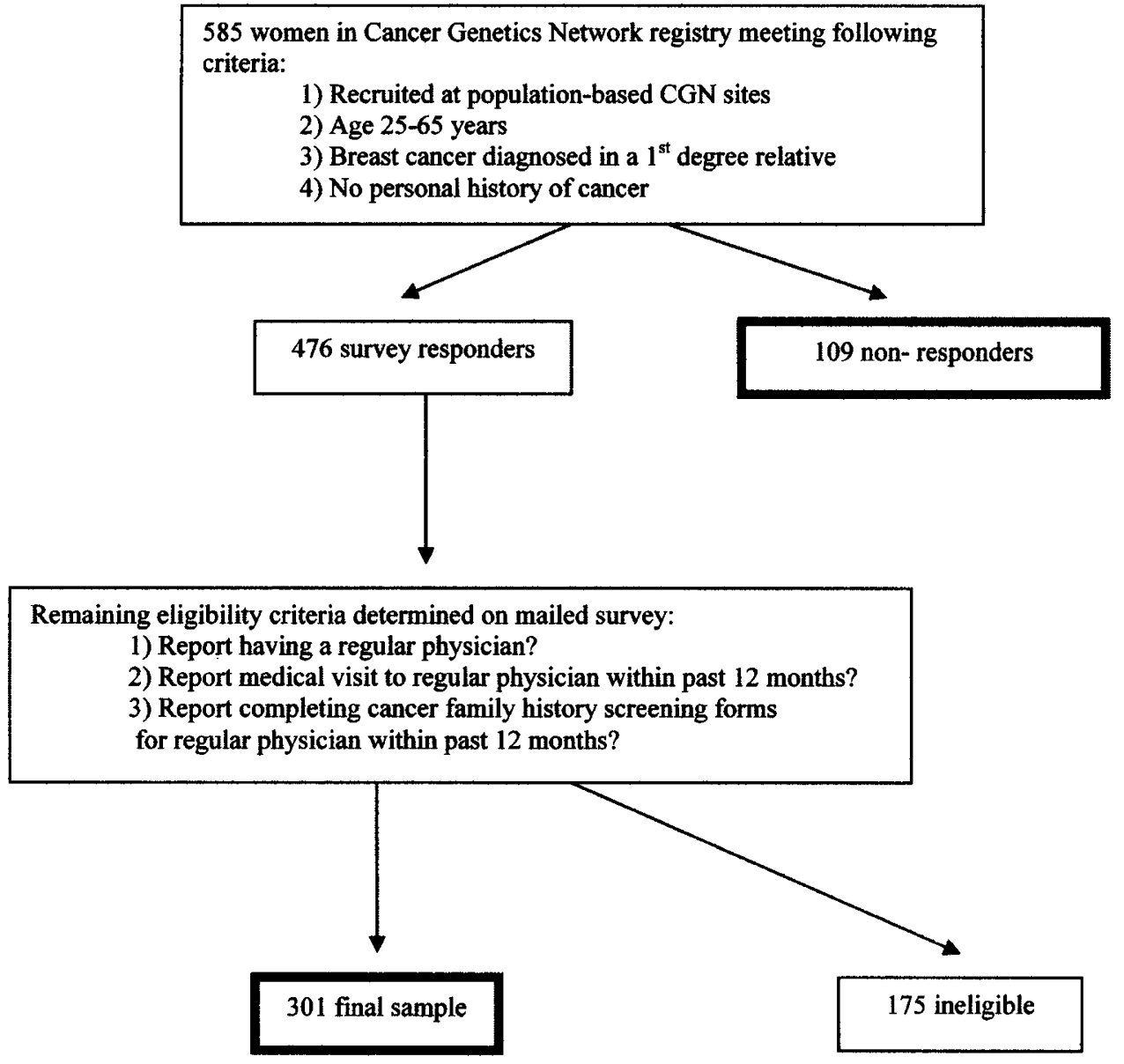

Fig. 1. Description of sample recruitment.

bility criteria of having and visiting a regular doctor within the past year and having their FMH collected in a manner consistent with screening procedures. We calculated the minimum response rate of seventy-three percent as standardized by the American Association for Public Opinion Research. ${ }^{16}$ This assumes that all of the 109 non-responders would have met final eligibility criteria and therefore is likely to substantially underestimate the true response rate. If we assume that the proportion of these 109 non-responders that would have met final eligibility criteria was the same as the proportion meeting eligibility criteria from the 476 initial responders (i.e., 301/476), then the approximated response rate would be eighty-two percent. Table 1 compares the study sample to non-responders. No statistically significant differences in mean age, education or family history characteristics were identified between these groups.

\section{Descriptive}

Table 1 presents the frequencies and distributions of all study variables. One out of three women reported direct genetic risk feedback from their regular doctor during the past year and $15(5 \%)$ reported a discussion about genetic referral.

\section{Main associations}

Table 2 presents crude and adjusted odds ratios for the relationship between reporting cancer risk feedback from one's regular doctor during the past year and one's self-rated breast cancer risk. Reporting any cancer risk feedback was associated with a greater odds of rating one's breast cancer risk to be "high" on both crude (OR: 2.53; 95\% CI: 1.41, 4.56) analyses and analyses adjusted for age, education, presence of multiple FDR's affected by breast cancer and early-onset breast cancer in a FDR (OR: 2.38, 95\% CI: 1.30, 4.38). The point estimate of association appeared strongest for feedback involving discussion of genetic referral/testing $\left(\mathrm{OR}_{\mathrm{adj}}\right.$ : $4.61,95 \% \mathrm{CI}$ : 0.57 , 37.2). However, the distribution and rarity of such feedback precluded valid quantitative assessment of this dimension of feedback in isolation (Table 2).

We observed an important degree of dose-dependent modification in our estimates of the relationship between feedback and self-rated risk by the degree of trust in one's regular doctor that ranged from an OR of 7.41 for those having greatest trust in their doctors to an OR of 1.05 for those with the least amount of trust in their doctors (Table 3 ).

\section{Other measures of risk perception}

Consistent with our hypothesis regarding the dimension of risk perception most effected by feedback following family history screening, only perceptions of risk involving the subjective classification of cancer risk as "high" were associated with the 
Table 1

Description of study sample

\begin{tabular}{|c|c|c|}
\hline Characteristic & $\begin{array}{c}\text { Study } \\
\text { sample } \\
(\mathrm{N}=301)\end{array}$ & $\begin{array}{c}\text { Non- } \\
\text { responders } \\
(\mathrm{N}=109)\end{array}$ \\
\hline Age mean (SD) & $46.9(10.5)$ & $45.2(13.3)$ \\
\hline \multicolumn{3}{|l|}{ Education } \\
\hline HS grad or less & $40(13 \%)$ & $14(13 \%)$ \\
\hline Some college & $107(36 \%)$ & $50(46 \%)$ \\
\hline College grad & $154(51 \%)$ & $45(41 \%)$ \\
\hline \multicolumn{3}{|l|}{ Higher-risk criteria: } \\
\hline Affected father or brother & $0(0 \%)$ & $1(1 \%)$ \\
\hline FDR with onset $\leq$ age 50 years & $140(47 \%)$ & $49(45 \%)$ \\
\hline Multiple affected FDR's & $52(17 \%)$ & $21(19 \%)$ \\
\hline Non-white & $7(2 \%)$ & $1(1 \%)$ \\
\hline Ashkenazi & & $1(1 \%)$ \\
\hline \multicolumn{3}{|l|}{ Feedback within last year } \\
\hline Direct risk communication & $98(33 \%)$ & \\
\hline Genetic counseling/testing & $15(5 \%)$ & \\
\hline Either & $101(34 \%)$ & \\
\hline \multicolumn{3}{|c|}{ "I am at high risk for getting breast cancer" } \\
\hline Strongly disagree & $10(3 \%)$ & \\
\hline Disagree & $40(13 \%)$ & \\
\hline Somewhat disagree & $38(13 \%)$ & \\
\hline Somewhat agree & $83(28 \%)$ & \\
\hline Agree & $79(26 \%)$ & \\
\hline Strongly agree & $49(16 \%)$ & \\
\hline
\end{tabular}

Table 2

Crude and adjusted estimates of association between regular physician feedback within past year and self-rated risk status

\begin{tabular}{|c|c|c|c|c|}
\hline & \multicolumn{2}{|c|}{$\begin{array}{l}\text { Endorse } \\
\text { risk status } \\
\text { as "high" }\end{array}$} & \multirow[b]{2}{*}{ OR $(95 \% \mathrm{CI})$} & \multirow[b]{2}{*}{$\mathrm{OR}_{\mathrm{adj}}(95 \% \mathrm{CI})$} \\
\hline & Yes & No & & \\
\hline Any feedback & 82 & 18 & $2.53(1.41,4.56)$ & $2.38(1.30,4.38)$ \\
\hline No feedback & 126 & 70 & - & - \\
\hline $\begin{array}{l}\text { Genetic referral } \\
\text { feedback }\end{array}$ & 14 & 1 & $6.14(0.80,47.46)$ & $4.61(0.57,37.25)$ \\
\hline $\begin{array}{l}\text { No genetic } \\
\text { referral } \\
\text { feedback }\end{array}$ & 196 & 86 & - & - \\
\hline $\begin{array}{l}\text { Direct risk } \\
\text { feedback }\end{array}$ & 79 & 18 & $2.38(1.32,4.29)$ & $2.30(1.25,4.23)$ \\
\hline $\begin{array}{l}\text { No direct risk } \\
\text { feedback }\end{array}$ & 129 & 70 & - & - \\
\hline
\end{tabular}

Logistic regression model: High risk $=$ feedback, age, education, multiple FDRs, early-onset breast cancer in FDR.
Table 3

Dose-response modification between feedback and self-rated risk by level of trust in one's physician

\begin{tabular}{lccc}
\hline & $\begin{array}{c}\text { Sample } \\
\text { size }\end{array}$ & $\begin{array}{c}\text { Reporting } \\
\text { feedback }(\%)\end{array}$ & $\begin{array}{c}\text { Association between } \\
\text { feedback and high } \\
\text { self-rated riskOR } \\
(95 \% \text { CI })\end{array}$ \\
\hline Very high trust & 67 & 43.9 & $7.41(1.35,40.74)$ \\
High trust & 59 & 34.5 & $4.72(1.07,20.82)$ \\
Moderate trust & 76 & 30.0 & $1.73(0.45,6.64)$ \\
Low trust & 95 & 30.6 & $1.05(0.38,2.95)$ \\
\hline
\end{tabular}

Logistic regression model: Self-rated "high" risk = feedback, age, education, multiple FDRs, early-onset breast cancer in FDR.

recall of feedback. When analyzed as a continuous scale measure, recalled feedback was strongly and positively associated with endorsement of being at "high risk" $(\beta=0.63, P<$ $0.0001)$. However, feedback was not significantly associated with either perceived likelihood $(\beta=0.17, P=0.14)$ nor perceived severity of developing cancer $(\beta=0.04, P=0.75)$. Similarly, when dichotomizing perceived likelihood to "likely" versus "unlikely" and perceived severity to "agree" and "disagree," no significant association with feedback emerged.

\section{DISCUSSION}

Our findings suggest that, among women with a first-degree family history of breast cancer, physician feedback arising from family history solicitation shapes women's categorization of their breast cancer risk to be "high." Furthermore, we observed that this relationship was influenced in a dose-dependent manner based on the woman's degree of trust in her doctor. These findings are consistent with physician-patient communication in primary care practice for three reasons related to physician handling of family history.

First, risk assessment is a large part of preventive care, and physicians frequently screen for a variety of conditions conferring risk to their patients. However, physicians and patients are faced with limited time during encounters in which to discuss the results of each risk assessment. This creates a triage context in which the physician often prioritizes those issues that she deems to be clinically important. Therefore, the occurrence of physician feedback is likely to indicate a context in which the physician has assessed the patient's familial breast cancer risk to be high.

Second, the direction of association (i.e., physician feedback promoting higher self-rated risk) is consistent with biases inherent to use of family history in clinical practice. Categorizing the clinical relevance of family cancer history is challenging given low levels of physician confidence in pedigree analysis ${ }^{3}$ and guidelines for establishing whether one's family history should trigger nonstandard approaches to breast cancer prevention are not uniform in their determination of high risk status. ${ }^{17}$ Given that substantial medico-legal pressures related to missed or delayed diagnoses of breast cancer impact primary 
care physicians, ${ }^{18,19}$ physicians harboring any ambiguity about their patient's family history of breast cancer are likely to favor more versus less aggressive recommendations about preventive interventions. For example, when a physician provides feedback to a young female patient having a first-degree family history of breast cancer, it is plausible that the doctor will consciously or subconsciously favor messages to their patients that reinforce overestimates rather than underestimates of breast cancer risk in order to avoid the downstream situation of "my doctor didn't tell me that I was at high risk, so I waited to get a mammogram."

Third is the specificity of the observed relation. We observed that the association between physician feedback and self-rated risk was evident when self-ratings of risk were based on categorizing oneself as being at "high risk" versus not. However, when participants rated their absolute likelihood of developing breast cancer, no such association emerged. Our measures for self-rated breast cancer risk assess different dimensions of risk judgments (absolute likelihood vs. membership in a "high risk" category) and cue participant's responses differentially. Therefore, we expect variability between responses to each item. For the relation under study here, we were most interested in those judgments that are likely to be influenced by physician feedback in the natural clinical setting. Prior work demonstrates that communicating in quasi-numeric terms about absolute risk is challenging ${ }^{12,20-22}$ and that physicians are predisposed to labeling risk groups in qualitative terms that reflect threshold of clinical importance (i.e., "high," "normal," "low") for the purposes of triage and decision-making. ${ }^{12}$ Therefore, our a priori hypothesis for this exploratory study was that physician feedback in the natural course of familial breast cancer risk assessment would influence the degree to which women labeled themselves as belonging to a "high risk" group. Our observations that the association is, in fact, specific to this dimension of perceived risk rather than ubiquitous across measures of perceived risk lend potentially important insights into the process by which physicians shape women's understanding of their personal risk. Longitudinal research is needed that directly observes patient-physician communication about inherited breast cancer risk in order to better understand this phenomenon.

We chose to restrict our sample to women whose family history was solicited in a systematic way (i.e., by office forms) to avoid the context of women presenting to their doctor with concerns or worries about their family history. Therefore, much of the feedback arising in this context is likely to be initiated by the doctor ("Given your family history, I'd recommend ...") rather than solicited by the patient. Of importance is that only one-third of women recall any feedback. Prior observation finds that solicitation of family history in primary care is associated with lower levels of worry about cancer. ${ }^{23}$ This study raises questions about whether the mechanism by which soliciting family history leads to reduced worry is via the absence of feedback to women reporting a family history. This "no news is good news" phenomenon is a frequent, and usually unintended form of inferred communication in clinical care.
This is an exploratory study with several limitations to causal inference. First, this is cross-sectional data collection of a recalled exposure. This context is prone to recall bias. We attempted to minimize recall bias by shortening the time frame in which we considered whether the physician provided feedback to the women to one year. Our study was designed to explore potential influences of physician feedback on women's self-ratings about breast cancer risk. However, it is plausible that our findings might represent reverse causality. For example, if women who perceive higher levels of breast cancer risk are more likely to recall physician feedback, then we might find our observed relationship. Furthermore, if higher levels of trust in one's physician also lead to differential recall of physician feedback, then this might be captured in our findings about the dose-dependent effects of trust on our main association. However, we did not observe higher frequencies of recalled physician feedback among women rating their risk as more likely versus less likely. Therefore, we believe that recall bias does not contribute substantially to our conclusions because our observed findings are specific to the dimension of risk judgments that are most likely to be influenced by physician approaches to risk feedback during clinical encounters.

Secondly, this is a registry-based study prone to sampling bias. We sought to minimize this bias by choosing to sample women from the population-based recruitment centers from the Cancer Genetics Network and not from the CGN sites that recruited women already accessing high-risk cancer centers. We expect that the direction of sampling bias in this study would underestimate the impact of feedback from one's doctor because the women in this study are more educated than the majority of primary care patients and have greater access to information about breast cancer genetics beyond that provided by their doctors.

Familial and genetic susceptibility to breast cancer is a major focus of societal messages to American women in the emerging Genomic Era. Primary care physicians handle the vast majority of triage with regards to which women should consider pursuing genetic testing or more aggressive cancer screening protocols. While feedback that women receive from their regular doctors is likely to be brief, the trust that is nurtured in many continuity-based medical relationships will make women likely internalize their doctor's messages, opinions and recommendations. Prior empirical work suggests that higher risk perceptions lead to higher levels of cancer-related worry ${ }^{24}$ and that both higher risk perceptions ${ }^{25}$ and higher levels of cancer worry ${ }^{26,27}$ predict mammography completion. However, extremes of cancer worry might also deter mammography ${ }^{28}$ and lead to health-related dysfunction. ${ }^{29}$ This study suggests that physician feedback influences self-ratings of breast cancer risk and that research regarding the psychosocial and behavioral impact of genetic testing for breast cancer susceptibility needs attention to the processes of triage at the primary care level. ${ }^{30}$

\section{ACKNOWLEDGMENTS}

The primary author thanks the Cancer Genetics Network Principal Investigators and their research teams for volunteer- 


\section{Gramling et al.}

ing the substantial resources necessary for completion of this study. The principal author was supported by a grant from the National Cancer Institute during the completion of this study (R25 CA87972-02).

\section{References}

1. United States Preventive Services Task Force. Genetic risk assessment and BRCA mutation testing for breast and ovarian cancer susceptibility. http://www.ahrq.gov/ clinic/uspstf/uspsbrgen.htm. Accessed March 10, 2006

2. United States Preventive Services Task Force. Screening for breast cancer. http:// www.ahrq.gov/clinic/uspstf/uspsbrca.htm. Accessed August 8, 2006.

3. Gramling R, Nash J, Siren K, Eaton C, et al. Family physician self-efficacy with screening for inherited cancer risk. Ann Fam Med 2004;2:130-132.

4. DiClemente RJ, Crosby RA, Kegler MC, et al. Emerging theories in health promotion practice and research. San Francisco: Jossey-Bass; 2002.

5. Glanz K, Rimer B, Lewis FM, et al. Health behavior and health education: Theory, research and practice. 3rd ed. San Francisco: Jossey-Bass; 2002.

6. Witte K, Cameron KA, McKeon JK, Berkowitz JM. Predicting risk behaviors: development and validation of a diagnostic scale. J Health Commun 1996;1:317-341.

7. Nelson N. Cancer Genetics Network gets under way with 5 years of funding. J Natl Cancer Inst 1997;89:10-11.

8. Senior K. New US cancer genetics network announced. Mol Med Today 1998;4:459-460.

9. Anton-Culver H, Ziogas A, Bowen D, Finkelstein D, et al. The Cancer Genetics Network: Recruitment results and pilot studies. Community Genet 2003;6:171-177.

10. Stange KC, Zyzanski SJ, Smith TF, Kelly R, et al. How valid are medical records and patient questionnaires for physician profiling and health services research? A comparison with direct observation of patients visits. Med Care 1998;36:851-867.

11. Safran DG, Kosinski M, Tarlov AR, Rogers WH, et al. The Primary Care Assessment Survey: Tests of data quality and measurement performance. Med Care 1998;36: 728-739.

12. Gramling R, Irvin JE, Nash J, Sciamanna C, et al. Numeracy and medicine: Key family physician attitudes about communicating probability with patients. $J$ Am Board Fam Pract 2004;17:473.

13. Weinstein ND. What does it mean to understand a risk? Evaluating risk comprehension. J Natl Cancer Inst Monogr 1999;25:15-20.

14. Diefenbach MA, Weinstein ND, O'Reilly J. Scales for assessing perceptions of health hazard susceptibility. Health Educ Res 1993;8:181-192.
15. Scheuner MT, Wang SJ, Raffel LJ, Larabell SK, et al. Family history: a comprehensive genetic risk assessment method for the chronic conditions of adulthood. Am J Med Genet 1997;71:315-324

16. The American Association of Public Opinion Research. Standard definitions: Final disposition of case codes and outcomes rates for surveys. 3rd edition. Lenexa, KS; 2004.

17. Palomaki GE, McClain MR, Steinort K, Sifri RD, et al. Screen positive rates and agreement among six family history screening protocols for breast/ovarian cancer in a population-based cohort of 21 to 55 year old women. Genet Med 2006; 8:161-168.

18. Frank-Stromborg M, Bailey LJ. Cancer screening and early detection: managing malpractice risk. Cancer Pract 1998;6:206-216.

19. Dewar MA, Love N. Legal issues in managing breast disease. Postgrad Med. Oct 1992;92:137-151

20. Edwards A, Elwyn G, Mulley A. Explaining risks: turning numerical data into meaningful pictures. BMJ 2002;324:827-830.

21. Lipkus IM, Samsa G, Rimer BK. General performance on a numeracy scale among highly educated samples. Med Decis Making 2001;21:37-44.

22. Woloshin S, Schwartz LM, Moncur M, Gabriel S, et al. Assessing values for health: numeracy matters. Med Decis Making 2001;21:382-390.

23. Gramling R, Anthony D, Lowery J, Ballinger L, et al. Association between screening family medical history in general medical care and lower burden of cancer worry among women with a close family history of breast cancer. Genet Med 2005;7:640 645 .

24. Lipkus IM, Klein WMP, Sugg Skinner C, Rimer BK. Breast cancer risk perceptions and breast cancer worry: what predicts what? J Risk Res 2005;8:439-452.

25. Vernon SW. Risk perception and risk communication for cancer screening behaviors: a review. J Natl Cancer Inst Monogr 1999:101-119.

26. Hay JL, McCaul KD, Magnan RE. Does worry about breast cancer predict screening behaviors? A meta-analysis of the prospective evidence. Prev Med 2006.

27. McCaul KD, Branstetter AD, O'Donnell SM, Jacobson K, et al. A descriptive study of breast cancer worry. J Behav Med 1998;21:565-579.

28. Andersen MR, Smith R, Meischke H, Bowen D, et al. Breast cancer worry and mammography use by women with and without a family history in a populationbased sample. Cancer Epidemiol Biomarkers Prev 2003;12:314-320.

29. Trask PC, Paterson AG, Wang C, Hayasaka S, et al. Cancer-specific worry interference in women attending a breast and ovarian cancer risk evaluation program: Impact on emotional distress and health functioning. Psychooncology 2001;10:349360. 